\title{
Hose Instability and Wake Generation by an Intense Electron Beam in a Self-Ionized Gas
}

\author{
S. Deng, ${ }^{1}$ C. D. Barnes,${ }^{3}$ C.E. Clayton, ${ }^{2}$ C. O'Connell,${ }^{3}$ F. J. Decker, ${ }^{3}$ R. A. Fonseca, ${ }^{2}$ C. Huang, ${ }^{2}$ M. J. Hogan, ${ }^{3}$ \\ R. Iverson, ${ }^{3}$ D. K. Johnson, ${ }^{2}$ C. Joshi, ${ }^{2}$ T. Katsouleas, ${ }^{1}$ P. Krejcik, ${ }^{3}$ W. Lu, ${ }^{2}$ W. B. Mori, ${ }^{2}$ P. Muggli, ${ }^{1}$ E. Oz, ${ }^{1}$ F. Tsung, ${ }^{2}$ \\ D. Walz, ${ }^{3}$ and M. Zhou ${ }^{2}$ \\ ${ }^{1}$ University of Southern California, Los Angeles, California 90089, USA \\ ${ }^{2}$ University of California, Los Angeles, California 90095, USA \\ ${ }^{3}$ Stanford Linear Accelerator Center, Menlo Park, California 94025, USA
}

\begin{abstract}
The propagation of an intense relativistic electron beam through a gas that is self-ionized by the beam's space charge and wakefields is examined analytically and with 3D particle-in-cell simulations. Instability arises from the coupling between a beam and the offset plasma channel it creates when it is perturbed. The traditional electron hose instability in a preformed plasma is replaced with this slower growth instability depending on the radius of the ionization channel compared to the electron blowout radius. A new regime for hose stable plasma wakefield acceleration is suggested.
\end{abstract}

Focused and compressed charged particle beams are the highest energy density drivers available in the laboratory. For example, a $50 \mathrm{GeV}$ electron beam from the Stanford Linear Accelerator Center (SLAC) can be focused to an intensity of a PW $/ \mu \mathrm{m}^{2}$. Such beams when interacting with matter invariably turn the matter to a plasma and exhibit a rich variety of ultrarelativistic beam-plasma phenomena including collective refraction [1], focusing [2], wakefield generation, and particle acceleration [3]. Many of these physical processes are expected to occur in astrophysical events such as supernova [4] and various astrophysical jets.

In this Letter we examine a new fundamental beamplasma instability that results when the beams become sufficiently intense that the gas/plasma is self-ionized by the beam. We refer to this as ionization hosing and analyze it with analytic and 3D particle-in-cell simulations models. We compare the results to the familiar electron hose instability in a preformed plasma [5] and discuss the implications for plasma wakefield accelerators. The instability of intense beams to hosing in preformed plasmas has been examined by several authors for particle [5,6] as well as laser [7-9] beams. In the traditional electron hose instability, a positive feedback occurs between a beam centroid oscillation and oscillations of plasma electrons that are kept out by the beam's space charge. In the instability considered here, a positive feedback occurs between a beam centroid oscillation and the displaced ion column it creates through ionization. The presence of an instability associated with self-ionization of a gas has been noted previously for the case of laser beams [10]. Here we take up the similar question for particle beams.

The mechanism for the transverse instability investigated here is illustrated in Fig. 1(a). A dense electron beam with space charge electric field exceeding the tunneling ionization threshold $[11,12]$ is propagating to the right. The head of the beam is below threshold for ionization and is unaffected (cyan). The later part of the beam (yellow) begins to ionize the gas, and the beam space charge expels the newly created electrons from the beam path. This creates a positively charged ion column. However, as a result of the tilt of the beam, the ion column is not symmetric about the beam (i.e., at a point above the beam axis the beam field has already passed through while at the same $x$ position below the beam the beam field has not yet arrived). Thus there is an offset between the central axis of the ion column and the beam axis. The displaced ion column creates a deflecting force on the beam that can lead to a positive feedback and to hosing of the beam.

The physical model above gives rise to a simple analytic description of ionization hosing. The beam centroid undergoes betatron oscillations caused by the focusing force of the ion column. The effect of a small displacement or offset of the column is an additional deflection force on the beam. The ion column centroid at any time takes the approximate form of the beam centroid position at a time earlier by an amount $\tau$ (on the order of the beam blowout time $1 / \omega_{\mathrm{pb}}$ where $\omega_{\mathrm{pb}}$ is the beam-plasma frequency defined by $\omega_{\mathrm{pb}}^{2}=$ $4 \pi n_{b} e^{2} / m$ and $n_{b}$ is the beam density at the point where the ionization threshold is reached). This is further justified by simulations [see Figs. 1(b) and 1(c)] discussed later. Thus the coupled equations for the beam and ion column centroids, $x_{b}$ and $x_{i}$, are

$$
\begin{gathered}
\partial_{s}^{2} x_{b}+k_{\beta}^{2} x_{b}=k_{\beta}^{2} x_{i} \\
x_{i}(\xi)=x_{b}(\xi+c \tau),
\end{gathered}
$$

where $k_{\beta}$ is the betatron frequency $\omega_{p} / \sqrt{2 \gamma} c$ [13], $s$ is the coordinate along the propagation direction (i.e., $s=z$ ), and $\xi$ is the coordinate measured form the head of the beam (i.e., $\xi=z-c t$ ). We note that these equations differ from the coupled equations for the electron hose instability in a preformed ion channel [5] by the form of Eq. (2).

These coupled equations can be solved by Fourier transforming in $\xi$ [e.g., $\overline{x_{b}(s, \omega)}=\frac{1}{\sqrt{2 \pi}} \int x_{b}(s, \xi) e^{i \omega \xi} d \xi$ where 
$\omega$ is the conjugate variable to $\xi$ ]. Combining the resulting equations gives

$$
\partial_{s}^{2} \bar{x}_{b}+k_{\beta}^{2} \bar{x}_{b}\left(1-e^{-i \omega c \tau}\right)=0 .
$$

Inverting the Fourier transform gives $x_{b}$ :

$$
x_{b}(s, \xi)=\frac{1}{\sqrt{2 \pi}} \int A e^{s\left[i k_{\beta}\left(1-e^{-i \omega c \tau}\right)^{1 / 2}-i \omega \xi / s\right]} d \omega .
$$

Here $2 A$ is the Fourier transform of the initial beam tilt function, i.e., $2 A=\frac{1}{\sqrt{2 \pi}} \int_{0}^{\infty} x_{b}(0, \xi) e^{i \omega \xi} d \xi$. Equation (4) can be solved by the method of steepest descents [14]. Asymptotic solution exists for a short bunch $\left(k_{\beta} s \gg \eta \equiv\right.$ $-\frac{\xi}{c \tau}$ ), where $\xi=0$ at the head of the beam and becomes negative when moved to the back of the beam) with an initial linear offset:

$$
\begin{aligned}
x_{b}(s, \xi) / x_{b}(0, \xi)= & \frac{1}{\sqrt{2 \pi}} \frac{1 /\left[\arctan \left(\sqrt{\frac{1-Y}{Y}}\right)\right]^{2}+[\ln (2 \sqrt{Y})]^{2}}{\eta\left(k_{\beta} s\right)^{1 / 2}[Y(1-Y)]^{1 / 4}}\left(\frac{k_{\beta} s}{2 \eta}\right)^{\eta} e^{\eta} \cos \left[k_{\beta} s \sqrt{1-Y}-\arctan \left(\sqrt{\frac{1-Y}{Y}}\right) \eta\right. \\
& \left.-\frac{\pi}{2}-\frac{5}{2} \arctan \left(\sqrt{\frac{1-Y}{Y}}\right)\right] .
\end{aligned}
$$

Here $Y=\left(\frac{\eta}{k_{\beta} s}\right)^{2}$. The dominant term is $\left(\frac{s}{\eta}\right)^{\eta}$ under the condition $k_{\beta} s \gg \eta$. This shows how the growth increases with normalized bunch length $\eta$. Thus the initial hosing grows rapidly over a few betatron periods $\left(2 \pi / k_{\beta}\right)$ with a characteristic hose wavelength of approximately $4 c \tau$ and a power law growth with propagation distance $s$. A sample
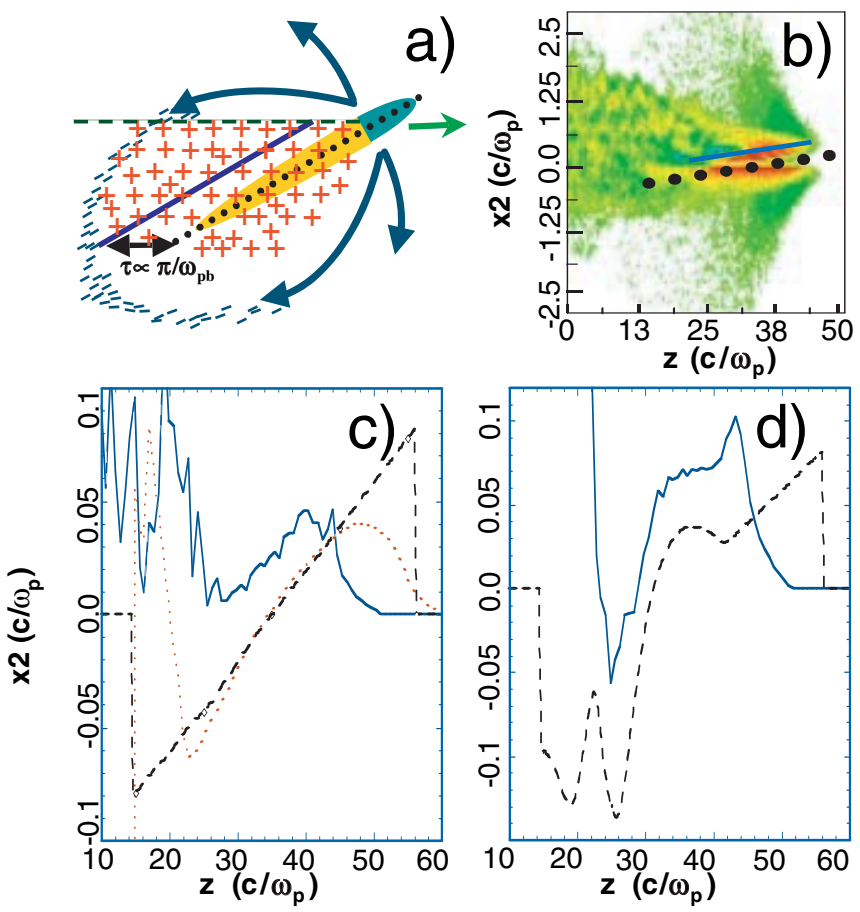

FIG. 1 (color). Mechanism of ionization hosing instability: (a) illustration of offset ion column formed by ionization of neutral gas by a tilted beam; (b) particle-in-cell simulation of total charge density ionized by tilted beam (center of charge is shown by a solid line; center of beam by dots); (c) lineout from simulation of beam centroid (dashed line), center of resulting charge density in the self-ionized case (solid line), and a preformed case (dotted line); (d) lineout from simulation of beam centroid (dashed line) and center of resulting charge density (solid line) at a later time $(s=0.35 \mathrm{~m})$. Simulations are in the above threshold regime. numerical solution is shown in Fig. 2 along with a comparison to the analytic solution. Also for comparison, the numerical solution for electron hosing in a preformed plasma from Ref. [5] is shown (dotted line, different scale).

We note that ionization hosing has a different wavelength in $\xi$, has a power law rather than exponential times power law growth in $s$, and is orders of magnitude smaller for this example. Once the hosing begins, the simple picture given by Eq. (2) rapidly breaks down at the back of the beam. Furthermore, the periodic focusing of the beam changes the blowout time $\tau$, further complicating the analysis. Thus we consider this as a guide to early response and turn to detailed particle-in-cell simulations to study the full development of the instability.

We perform 3D particle-in-cell (PIC) simulations with the fully explicit code OSIRIS $[15,16]$ for both self-ionized and preionized plasma cases. The ionization algorithm used in OSIRIS is described in Ref. [16]; it includes both tunnel and impact ionization, but impact ionization is negligible for the example here. In order to illustrate the hosing, we choose beams a few plasma periods long, slightly longer than that would be used in a wakefield accelerator. The beam has a Gaussian shape with $\sigma_{z}=2520 \mu \mathrm{m}$, and $\sigma_{r}=70 \mathrm{~mm}$, an emittance of $15 \pi \mathrm{mm}$ mrad, total charge of $1.8 \times 10^{10}$ electrons at $30 \mathrm{GeV}(\gamma=60000)$, and a peak density of $n_{b}=1.75 n_{0}$, where $n_{0}$ is the density of plasma (for preionized case) or neutral gas density (for self-ionized case). The simulation

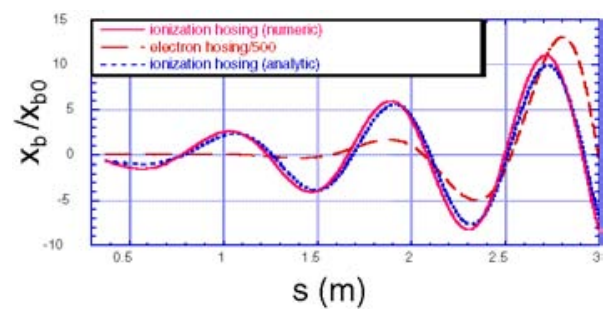

FIG. 2 (color online). Comparison of analytic and numerical solutions to Eq. (1) and (2) - beam centroid growth vs propagation distance at position $\xi=10.08 \mathrm{~mm}$ in the beam. 
system is a $70.4 \times 6 \times 6 \mathrm{c} / \omega_{p}$ box with metallic boundaries, containing $352 \times 60 \times 30$ cells and 16 particles per cell. The gas ionization rate $[11,12,17]$ used was $W\left[s^{-1}\right] \approx$ $3.46 \times 10^{21} \exp \left(-\frac{a}{E[G V / m]}\right) E^{5}$, where $a$ is related to gas ionization potential. We use the values for $a($ ?) of 1.5 , 0.8 , and 0.04 corresponding to near threshold, above threshold, and very far above threshold cases, respectively. These values are chosen to facilitate the comparison of physics of each of these regimes. Two recent plasma wakefield accelerator experiments at the SLAC known as E-164 [18] and E-164X [19] correspond to near threshold and far above threshold cases, respectively. Also for far above threshold case, the beam charge amount is 3 times larger. Figure 1(b) shows the real space at an early time $(s=$ $0.035 \mathrm{~m}$ ) of the electrons and ions tunnel ionized by a tilted beam propagating through a neutral gas. The tilt of the beam and the resulting ion column are more apparent from the lineout of the centroid of the ionized charge density and the beam in Fig. 1(c). Note that the two centroid curves are parallel over a significant portion of the beam but the charge centroid is displaced in $z$ by approximately $1.1 \mathrm{c} / \omega_{\mathrm{pb}}$. As shown in Fig. 1(c), this displacement does not occur for the preformed plasma case (represented by the dotted line). Figure 1(d) shows that the tilt of the resulting ion column still follows the tilt of the beam even after the beam starts hosing.

Figure 3 shows the later time development of hosing of the beam in three cases: (a) preformed plasma, (b) tunnelionized gas with a higher ionization potential (i.e., beam field is near ionization threshold - gas ionization width is less than beam blowout radius), and (c) tunnel-ionized gas with a low ionization potential (i.e., beam field is above ionization threshold - the gas ionization width is comparable to beam blowout radius). The results in Fig. 3(a) show the classic electron hose instability of Ref. [5]. The top solid curve is a plot of beam centroid versus $s$ at $\xi=$ $10.08 \mathrm{~mm}$, the middle image is a snapshot of the beam at $s=1.4 \mathrm{~m}$, and the bottom image is the corresponding plasma electron density. Figure 3(b) is the same beam in a self-ionized gas near the ionization threshold. We see that the hosing is greatly suppressed in this case. Through the simulations, we find that the electron hosing is suppressed because there are not enough plasma ions created to prevent the ionized electrons from leaving vertically due to blowout by the beam space charge (i.e., a surrounding electron channel never forms). This nonreturning blowout is apparent in the real space of plasma electrons shown in Fig. 3(b), bottom. We comment that the growth in Fig. 3(b) is even less than that predicted by our ionization hosing model. One reason for this is the effect of plasma dynamics on turning off the ionization midway through the beam. The beam field becomes partially neutralized as a result of electron blowout and the remaining ions. Once the ionization turns off, of course, the ionization hosing ceases. At the tail of the beam, the beam charge drops and the positive
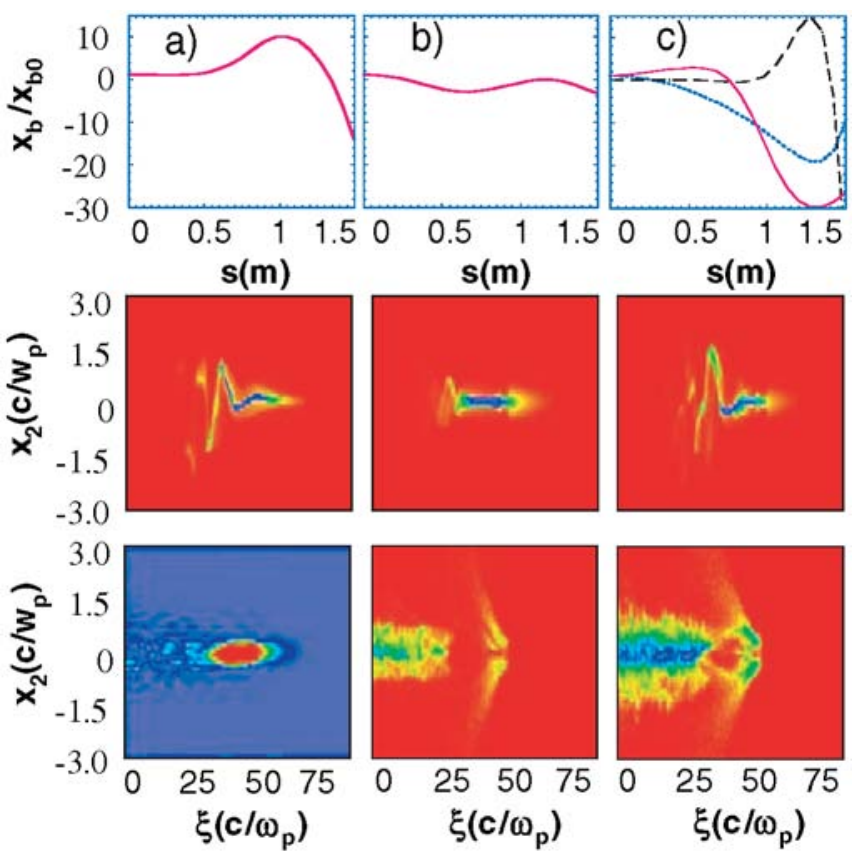

FIG. 3 (color). Particle-in-cell simulation results showing centroid of beam vs propagation distance (top), beam real space at $1.4 \mathrm{~m}$ (middle), and plasma electron density (bottom) for (a) preformed plasma, (b) near threshold, and (c) above threshold. Also shown on (c) is the unscaled numerical solution (dotted line) of the ionization hosing model Eqs. (3) and (4) with $t=$ $0.5 c / \omega_{\mathrm{pb}}-3 c / \omega_{p}$. The traditional hosing theory result (dashed line) is scaled by a factor of 500 to fit on the graph.

ions field becomes large enough to ionize more gas. This causes ionization hosing at the tail of the beam. Figure 3(c) shows the same beam in a gas with a low ionization threshold. When, as in this case, the gas ionization region is a little bit wider than the beam blowout region, then both ionization hosing and electron hosing mechanisms are present. As apparent from the bottom panel in Fig. 3(c), there are electrons that blow out and never return and other electrons that return. The nonreturning electrons create the ion charge asymmetry responsible for ionization hosing while the returning electrons form a channel for traditional hosing. The hosing in Fig. 3(c) is even more severe than in the preformed case. We attribute this to the added effect of ionization hosing. When the beam is very far above ionization threshold (not shown), the plasma response and wakes similar to that in a preformed plasma [12], and the hosing instability is similar to Fig. 3(a). Empirically, we find from simulations that these different regimes are separated by a condition that the blowout radius of electrons $r_{b}$ is equal to the radius of ionized gas $r_{i}$. Since the blowout radius is given by $r_{b} \propto 2 \sqrt{\frac{n_{b}}{n_{0}}} \sigma_{r}$, we can define the following condition for being in the regime where electron hosing is suppressed and ionization hosing occurs: $E_{b}>$ $E_{i}>\frac{E_{b}}{2 \sqrt{\frac{\pi_{b}}{n_{0}}}}$, where $E_{b}$ is the peak radial field of the beam 

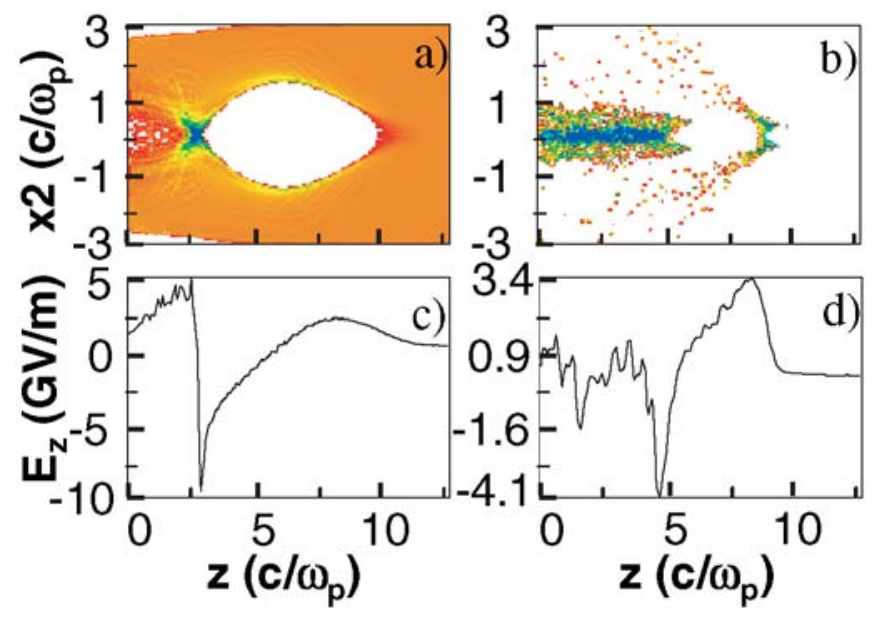

FIG. 4 (color). Particle-in-cell simulation comparisons of wakes in preformed plasma (left) and self-ionized plasma near threshold (right). (a) and (b) Real space of plasma electrons; (c) and (d) wake electric field $E_{z}$ on axis. Note that the blowout radius in (a) is clearly larger than the ionization radius in (b). $N_{\text {beam }}=2 \times 10^{10}, n_{0}=5 \times 10^{15}, \gamma=60000$.

$\left(E_{b} \propto 2 \pi n_{b} e \sigma_{r}\right)$ and $E_{i}$ is the approximate electric field threshold for ionizing the gas.

Next we examine the wakefields generated in the near threshold regime that is stable to hosing. To produce large wakes we shorten the beam to $100 \mu \mathrm{m}$, and narrow it to $12.5 \mu \mathrm{m}$. The other parameters are given in the caption to Fig. 4. Figure 4 shows the plasma real space and lineout of the axial wakefield $E_{z}$ at $z=0.92 \mathrm{~cm}$ for preformed and self-ionized plasmas. Figure 4(a) shows the classic electron blowout and return wakefield regime. Figure 4(b) shows that the electron response is completely different in the self-ionized case. The plasma electrons created at the head of the beam are blown out with such momentum that the amount of ion charge is insufficient to recapture them (i.e., they exceed escape velocity). The electron expulsion and remaining ions reduce (partially neutralize) the space charge electric field of the beam, turning off further ionization by the beam. However, once the beam leaves, the ions that remain are un-neutralized and their bare field is suddenly exposed and ionizes additional gas (at larger radius than the initial beam ionized). The newly ionized electrons see the large attractive potential of the ions and rush toward the axis, creating a large accelerating fieldapproximately half of that in the preionized case [Figs. 4(c) and $4(\mathrm{~d})]$.

In conclusion, we identified a new hosing mechanismionization hosing — and presented a simple analytical model for this new instability. Simulations suggest that the new instability replaces the traditional electron hose instability if the beam field is near the ionization threshold. A new two-stage wake generation mechanism is studied for the near threshold regime, in which the traditional electron hosing is eliminated while ionization hosing is suppressed. An example that is in this regime is the E-164 experiment at SLAC in its long bunch configuration $\left(s_{z}=100 \mu \mathrm{m}\right.$, $N=2 e 10, \sigma_{r}=15 \mu \mathrm{m}$ in Li gas at $5 \times 10^{15} \mathrm{~cm}^{-3}$ as in Fig. 4). Since the accelerating phase of the wake in that case is fully behind the beam, a separate beam load is needed to test for acceleration in that experiment. This near threshold regime may provide a way to overcome hose instability while providing large accelerating fields for future plasma wakefield accelerators.

The authors gratefully acknowledge the supercomputing time provided by the Center for High Performance Computing and Communications (HPCC) at USC (University of Southern California) and NERSC. This work is supported by U.S. Department of Energy, under Contracts No. USDOE DE-FG03-92ER40745, No. NSF-PHY0078715, No. DE-FC02-01ER41192, No. DE-FG0392ER40727, No. DE-FC02-01ER41179, No. PHY0078508, and No. DE-AC03-76SF00515.

[1] P. Muggli et al., Nature (London) 411, 43 (2001).

[2] J. S. T. Ng et al., Phys. Rev. Lett. 87, 244801 (2001).

[3] P. Muggli et al., Phys. Rev. Lett. 93, 014802 (2004).

[4] K. G. McClements, M.E. Dieckmann, A. Ynnerman, S. C. Chapman, and R. O. Dendy, Phys. Rev. Lett. 87, 255002 (2001).

[5] E. S. Dodd et al., Phys. Rev. Lett. 88, 125001 (2002).

[6] D. H. Whittum, W. M. Sharp, S.S. Yu, M. Lampe, and G. Joyce, Phys. Rev. Lett. 67, 991 (1991).

[7] G. Shvets and J.S. Wurtele, Phys. Rev. Lett. 73, 3540 (1994).

[8] Early work on so-called resistive hose instabilities were in a different regime of long beams in plasmas for which plasma inertia and ionization times were neglected. See, for example, E. P. Lee, Phys. Fluids 21, 1327 (1978).

[9] P. Sprangle, J. Krall, and E. Esarey, Phys. Rev. Lett. 73, 3544 (1994).

[10] T. M. Antonsen, Jr. and Z. Bian, Phys. Rev. Lett. 82, 3617 (1999).

[11] M. V. Ammosov, N.B. Delone, and V.P. Krainov, Sov. Phys. JETP 64, 1191 (1986).

[12] David L. Bruhwiler et al., Phys. Plasmas 10(5), 2022 (2003).

[13] C. E. Clayton et al., Phys. Rev. Lett. 88, 154801 (2002).

[14] G. B. Arfken and H.J. Weber, Mathematical Methods for Physicists (Academic, New York, 2001), 5th ed., pp. 477484.

[15] R. Hemker et al., Proceedings of the IEEE Particle Accelerator Conference, New York, 1999 (IEEE, Piscataway, NJ, 1999), Vol. 5, p. 3672, Catalog No. CH36366.

[16] S. Deng et al., AIP Conf. Proc. 647, 592 (2002).

[17] S. Deng et al., Phys. Rev. E 68, 047401 (2003).

[18] C. O'Connell, Phys. Rev. ST Accel. Beams 5, 121301 (2002).

[19] M. J. Hogan et al., Phys. Rev. Lett. 95, 054802 (2005). 